\title{
LOW $\mathrm{P}_{\mathrm{T}}$ HADRONIC PHYSICS WITH CMS
}

\author{
FERENC SIKLÉR \\ KFKI Research Insitute for Particle and Nuclear Physics \\ Konkoly-Thege Miklós út 29-33, Budapest, H-1121, Hungary \\ sikler@rmki.kfki.hu
}

for the CMS Collaboration

Received (22 February 2007)

Revised (revised date)

\begin{abstract}
The pixel detector of CMS can be used to reconstruct very low $p_{T}$ charged particles down to about $0.1 \mathrm{GeV} / c$. This can be achieved with high efficiency, good resolution and a negligible fake rate for elementary collisions. In the case of central $\mathrm{PbPb}$ collisions the fake rate can be kept low for $p_{T}>0.4 \mathrm{GeV} / c$. In addition, the detector can be employed for identification of neutral hadrons (VOs) and converted photons.
\end{abstract}

\section{Introduction}

The reconstruction of low $p_{T}$ charged and neutral hadrons (yields, spectra and correlations) is crucial to characterize the collective properties of the system produced in nucleus-nucleus collisions at the LHC. In pp collisions, the measurement of high $p_{T}$ observables also requires good understanding of the characteristics of the underlying event and backgrounds which are dominated by soft $p_{T}$ spectra 1 .

In CMS, the measurement of charged particle trajectories is achieved primarily using the silicon tracker with both pixels and strips, embedded in a $4 \mathrm{~T}$ magnetic field, and with geometric coverage over $|\eta|<2.5$. The high granularity silicon pixel tracker consists of three barrel layers (at about 4,7 and $11 \mathrm{~cm}$ radius) and two endcap disks. There are about 66 million pixels with an area of $100 \times 150 \mu \mathrm{m}^{2}$ and a sensor thickness of $300 \mu \mathrm{m}$. The strip part is a combination of single- and double-sided layers with ten barrel and nine forward layers on each side (9.3 million channels). The silicon tracker has excellent reconstruction performance for $p_{T}>1$ $\mathrm{GeV} / c: 95 \%$ efficiency for charged hadrons with high $p_{T}$, better than $98 \%$ for muons in pp and $\mathrm{pA}$ collisions and around $75 \%$ for central $\mathrm{PbPb} 2$.

The reconstruction capabilities at lower $p_{T}$ are limited by the high magnetic field and effects of the detector material. In addition, in central AA collisions the high occupancy of the silicon strips makes the inclusion of these strips in charged particle tracking difficult 2 . Using only silicon pixels allows the same analysis to be used for low multiplicity pp, pA and high multiplicity AA events. 

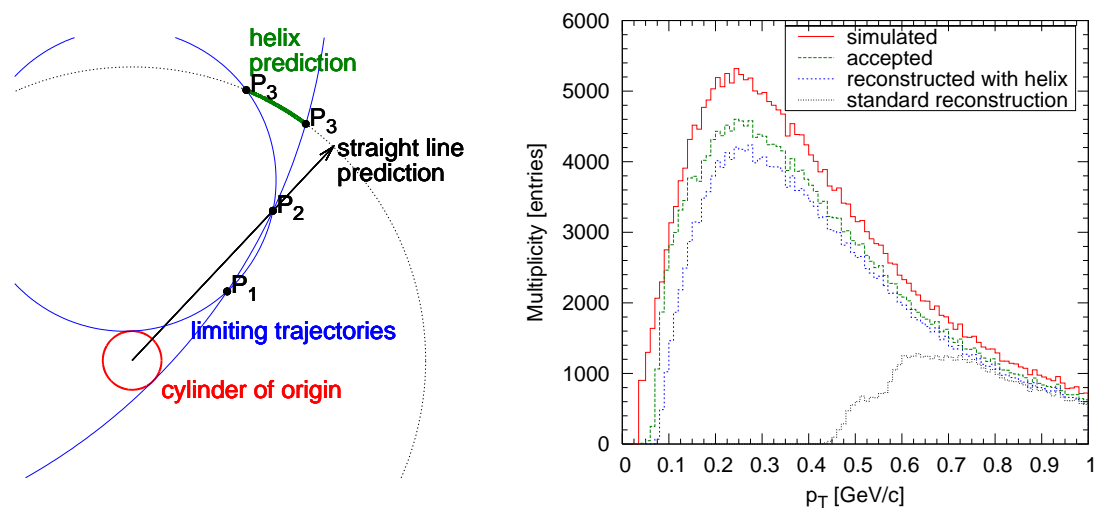

Fig. 1. Left: Schematic comparison of the standard straight line prediction and the new helix prediction for finding the third hit. Right: Transverse momentum distributions of the charged particles: simulated (solid red), accepted (green dashed) and reconstructed, with the standard method (dotted black) or with the new helix method (dotted blue).

\section{Track reconstruction}

We have developed an improved tracking algorithm which reconstructs tracks down to $0.1 \mathrm{GeV} / c$, using just the three pixel layers, with the modified hit triplet finding and cleaning procedures described here.

\subsection{Modified hit triplet finding}

The track finding procedure starts by pairing two hits from different layers (see Fig. 11). During the search for the third hit, the following requirements must be fulfilled: the track must come from the cylinder of origin (given by its radius, halflength and position along the beam-line); the $p_{T}$ of the track must be above the minimal value $p_{\mathrm{T}, \min }$; and the track must be able to reach the layer where the third hit may be located. In the small volume of the pixel detector the magnetic field is practically constant and the charged particles propagate on helices. The projection of a helix or a cylinder onto the transverse plane is a circle. Each requirement defines a region of allowed track trajectories. They are enclosed by a pair of limiting circles which can be constructed using simple geometrical transformations. A third hit candidate is accepted if its position is within a region which takes into account the expected multiple scattering. More details are given in Ref. 3 .

\subsection{Triplet cleaning}

While high $p_{T}$ tracks are relatively clean, uncorrelated hit clusters can often be combined to form fake low $p_{T}$ tracks. However, a cluster contains more information than its position. The geometrical shape of the hit cluster depends on the angle of incidence of the particle: bigger angles will result in longer clusters. We can, thus, 

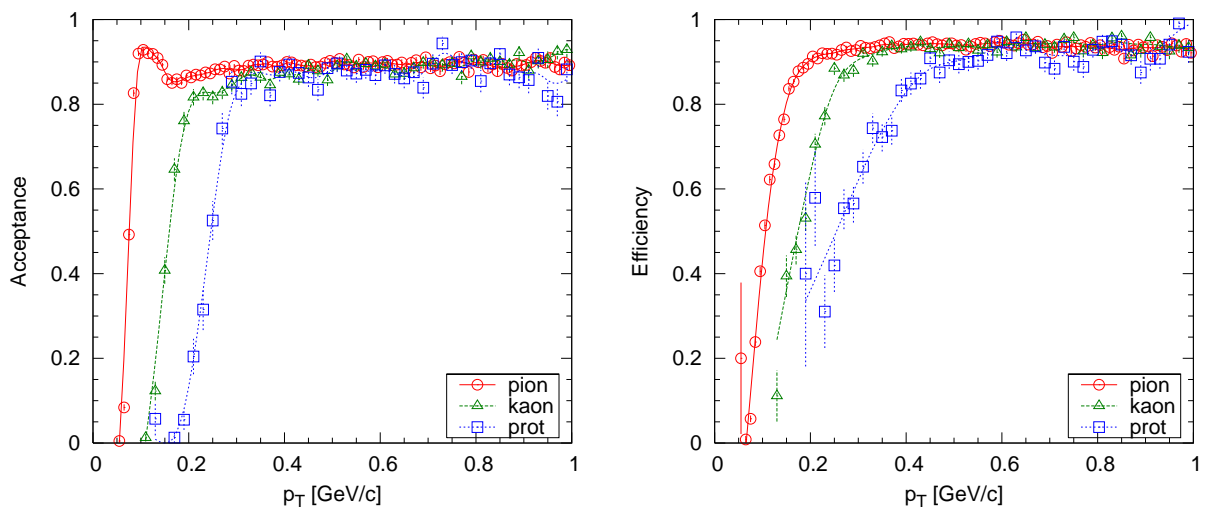

Fig. 2. Acceptance (left) and reconstruction efficiency (right) as a function of $p_{T}$, for tracks in the range $|\eta|<1$, for pions (circles), kaons (triangles) and (anti)protons (squares).

check whether the measured shape of the cluster is compatible with the predicted angle of incidence of the track; if any of the hits in the triplet is not compatible, the triplet is removed from the list of track candidates.

\subsection{Low $p_{T}$ tracking results}

The low $p_{T}$ reconstruction studies are based on 25000 minimum bias pp events (generated with Pythia, with the default minimum bias settings), reconstructed with the modified hit triplet finding. The algorithm uses the standard CMS settings, except for a much lower minimum $p_{T}(0.075 \mathrm{GeV} / c)$.

The acceptances rise sharply with $p_{T}$ (see Fig.2-left), and become approximately flat above $p_{T}$ values around $0.1,0.2$ and $0.3 \mathrm{GeV} / c$, respectively for pions, kaons and protons. In the range $|\eta|<2$, their averages are 0.88 (pions), 0.85 (kaons) and 0.84 (protons). Also the reconstruction efficiencies rise sharply with $p_{T}$ (see Fig. 2 . right), and become nearly flat above $p_{T}$ values around $0.2,0.3$ and $0.4 \mathrm{GeV} / c$, respectively for pions, kaons and protons. In the range $|\eta|<1.5$, the corresponding average reconstruction efficiencies are 0.90, 0.90 and 0.86 .

Without triplet cleaning, the fake rate is $\sim 4 \%$ at $\eta \sim 0$ and reaches $20 \%$ at $|\eta| \sim 2$. With cleaning, the fake rate decreases very significantly (by a factor of 10), to around $0.5 \%$ and $2 \%$ at $\eta \sim 0$ and $\sim 2$, respectively. In the range $|\eta|<1$, the fake rate decreases steeply with $p_{T}$, being about $4 \%$ at $0.1 \mathrm{GeV} / c, \sim 1 \%$ at $0.16 \mathrm{GeV} / c$ and at the per mil level for higher $p_{T}$ values.

Figure 3-left shows, as a function of the generated $p_{T}$ and separately for pions, kaons and protons, the ratio between the reconstructed and the simulated $p_{T}$ ("bias"). It is seen that the particles generated at low $p_{T}$ tend to be reconstructed with a slightly lower $p_{T}$ value, because of energy loss effects. This bias is negligible for high $p / m$ values but is quite significant for low momentum protons (or antiprotons): a correction of almost $10 \%$ is needed for protons of $p_{T} \sim 0.2 \mathrm{GeV} / c$. 

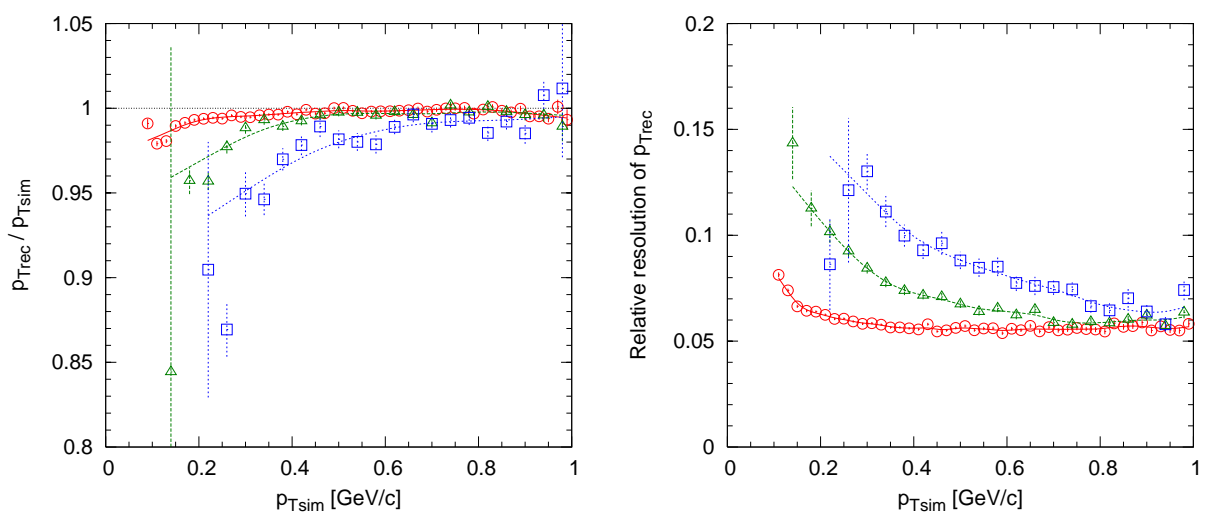

Fig. 3. Degradation of the reconstructed $p_{T}$ as a function of the simulated $p_{T}$, in terms of "bias" (left) and resolution (right), for pions (circles), kaons (triangles) and (anti)protons (squares), in the range $|\eta|<1$.

Figure 3-right shows how the resolution of the reconstructed $p_{T}$ depends on the generated $p_{T}$. While at high $p_{T}$ values the resolution is $\sim 6 \%$ for all particles, at low $p_{T}$ the multiple scattering and energy straggling effects are more important and lead to significantly degraded resolutions, in particular for protons.

The performance of the low $p_{T}$ reconstruction was studied under several running conditions. These studies are based on 25000 minimum bias pp events (Pythia generator) and on 25 central $\mathrm{PbPb}$ events (Hydjet generator) with two multiplicity settings: total particle multiplicities 30000 ("central") and 15000 ("mid-central"). In the $\mathrm{PbPb}$ case, the primary vertex of the event was determined first, with good precision, using high $p_{T}$ tracks. In a second step, the cylinder of origin was centered on this vertex, with a small half-length of $0.1 \mathrm{~cm}$. In order to further reduce the reconstruction rate of fake tracks, the radius of the cylinder of origin was reduced to $0.1 \mathrm{~cm}$. The reconstruction was made faster by increasing the minimum $p_{T}$ cut to $0.175 \mathrm{GeV} / c$.

The reconstruction efficiency is shown in Fig. 4- left, for pions, as a function of $p_{T}$. Above $p_{T}$ around $0.4 \mathrm{GeV} / c$, the pion reconstruction efficiency in $\mathrm{PbPb}$ collisions is $\sim 90 \%$, only $5 \%$ smaller than in pp collisions. Figure 4 right shows that the reconstruction rate of fake tracks falls steeply with increasing $p_{T}$. It drops below $10 \%$ for $p_{T} \sim 0.2 \mathrm{GeV} / c$ in high-luminosity pp collisions and for $p_{T} \sim 0.4 \mathrm{GeV} / c$ in central $\mathrm{PbPb}$ collisions.

\section{Neutral hadron (V0) and (converted) photon identification}

It was shown in the previous section that silicon detectors can detect charged particles with good position and momentum resolution. Some weakly-decaying neutral particles (V0s) such as $\mathrm{K}_{\mathrm{S}}^{0}, \Lambda$ and $\bar{\Lambda}$, have a sizeable probability to decay far from the primary event vertex $\left(c \tau=2.68\right.$ and $7.89 \mathrm{~cm}$ for $\mathrm{K}_{\mathrm{S}}^{0}$ and $\Lambda$, respectively). Likewise, 

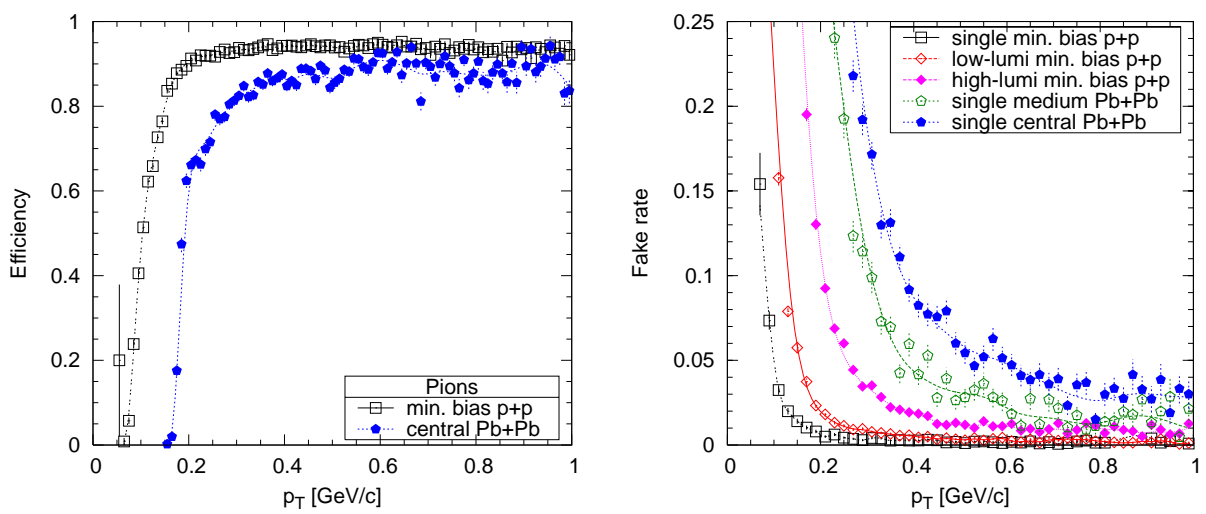

Fig. 4. Left: Pion reconstruction efficiency as a function of $p_{T}$ for tracks in the range $|\eta|<1$, for minimum bias pp events (squares) and for central $\mathrm{PbPb}$ collisions (circles). Right: Reconstruction rate of fake tracks as a function of $p_{T}$, for tracks in the range $|\eta|<1$, for single, low luminosity and high luminosity minimum bias pp events, and for central and mid-central $\mathrm{PbPb}$ collisions.

the silicon detectors can be used to reconstruct photons through their conversion to $\mathrm{e}^{+} \mathrm{e}^{-}$pairs in the material of the beam-pipe, silicon pixels and supports.

The analysis presented here only uses charged particles reconstructed from pixel hit triplets, with a much wider cylinder of origin $(3.0 \mathrm{~cm})$. Therefore, only neutral particles which decay up to the first pixel barrel layer can be found. Considering their masses and $p_{T}$ distributions, about half of the produced $\mathrm{K}_{\mathrm{S}}^{0}$ and $\Lambda$ particles satisfy this condition.

The search for V0 candidates reduces to the determination of the closest point between two helices, as described in detail in Ref. 3 . The distribution of the distance between the decay vertex and the beam-line $(r)$ is shown in Fig. 5-left. The $r$ distributions for V0s show an exponential behaviour, steeper for $\mathrm{K}_{\mathrm{S}}^{0}$ than for $\Lambda$, reflecting their different $c \tau$ values. The $r$ distribution for photons is completely different: the two peaks belong to the inner and outer silicon wafers of the first pixel barrel layer.

\subsection{VO results}

These studies are based on 25000 single minimum bias $\mathrm{p}+\mathrm{p}$ events (Pythia generator), reconstructed with the modified hit triplet finding. The invariant mass distribution of reconstructed $\mathrm{K}_{\mathrm{S}}^{0} \rightarrow \pi^{+} \pi^{-}$decays is shown in Fig. 55-right. The $\mathrm{K}_{\mathrm{S}}^{0}$ is reconstructed with a resolution of $16 \mathrm{MeV} / c^{2}$, with an average mass of $0.496 \mathrm{GeV} / c^{2}$, in agreement with the nominal mass value. The $\Lambda$ and $\bar{\Lambda}$ peaks (not shown) are located at $1.114 \mathrm{GeV} / c^{2}$, with a resolution of $6 \mathrm{MeV} / c^{2}$. Protons can be strongly enhanced by a cut on the truncated mean of their $\mathrm{d} E / \mathrm{d} x$, removing almost all the background. In the case of single collisions or low-luminosity pp running, the resonances can be exclusively identified. For high-luminosity pp running 

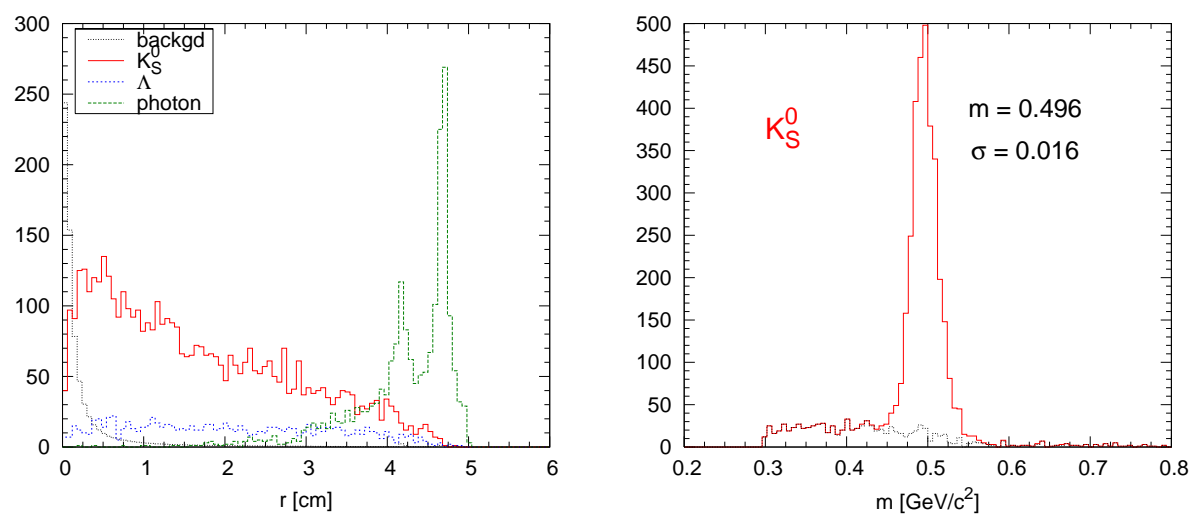

Fig. 5. Left: Distribution of the distance $(r)$ between the "decay vertex" and the beam-line for $\mathrm{K}_{\mathrm{S}}^{0}$, $\Lambda$, converted photons and background (fake particle pairs). Right: Invariant mass distribution of reconstructed $\mathrm{K}_{\mathrm{S}}^{0} \rightarrow \pi^{+} \pi^{-}$. The mass distribution of the background (fake pion pairs) is indicated by the dashed line. The results of a Gaussian fit to the signal are given in units of $\mathrm{GeV} / c^{2}$.

or $\mathrm{PbPb}$ collisions, the inclusive yield can still be extracted, with a relatively small background.

\section{Conclusions}

With a modified hit triplet finding algorithm, the pixel detector can be employed for the reconstruction of low $p_{T}$ charged hadrons in high luminosity pp collisions, as well as in $\mathrm{PbPb}$ reactions. The acceptance of the method extends down to 0.1, 0.2 and $0.3 \mathrm{GeV} / c$ in $p_{T}$ for pions, kaons and protons, respectively. The fake track rate can be greatly reduced by using the geometrical shape of the pixel clusters. Weakly-decaying hadrons $\left(\mathrm{K}_{\mathrm{S}}^{0}, \Lambda\right.$ and $\left.\bar{\Lambda}\right)$ decaying before the first pixel layer can be observed via their charged products. Photons converting in the beam-pipe or in the first pixel barrel layer are also detectable.

In summary, the CMS detector is able to provide good quality data on low $p_{T}$ charged and neutral particle spectra and yields, thus contributing to the soft hadronic physics program at the LHC.

\section{Acknowledgements}

The author is thankful to David d'Enterria, Carlos Lourenço and other members of the CMS Heavy Ion group for their valuable comments and corrections to the text. This work was supported by the Hungarian Scientific Research Fund (T 048898).

\section{References}

1. J. P. Revol [ALICE Collaboration], Eur. Phys. J. directC 4S1 (2002) 14 [Pramana 60 (2003) 795].

2. Christof Roland, CMS Note 2006/031.

3. "CMS Physics TDR Addendum: High Density QCD with Heavy-Ions", CERN-LHCC2007, to be submitted. 